\title{
Dust reverberation-mapping of the Seyfert 1 galaxy WPVS48 ${ }^{\star}$
}

\author{
F. Pozo Nuñez ${ }^{1}$, M. Haas ${ }^{1}$, R. Chini ${ }^{1,2}$, M. Ramolla ${ }^{1}$, C. Westhues ${ }^{1}$, K. Steenbrugge ${ }^{2,3}$, L. Kaderhandt ${ }^{1}$, H. Drass ${ }^{1}$, \\ R. Lemke ${ }^{1}$, and M. Murphy ${ }^{4}$ \\ 1 Astronomisches Institut, Ruhr-Universität Bochum, Universitätsstraße 150, 44801 Bochum, Germany \\ 2 Instituto de Astronomia, Universidad Católica del Norte, Avenida Angamos 0610, 1280 Casilla Antofagasta, Chile \\ 3 Department of Physics, University of Oxford, Keble Road, Oxford OX1 3RH, UK \\ 4 Departamento de Física, Universidad Católica del Norte, Avenida Angamos 0610, 1280 Casilla Antofagasta, Chile
}

Received 3 December 2013 / Accepted 16 December 2013

\section{ABSTRACT}

\begin{abstract}
Using robotic telescopes of the Universitätssternwarte Bochum near Cerro Armazones in Chile, we monitored the $z=0.0377$ Seyfert1 galaxy WPVS48 (2MASX J09594263-3112581) in the optical $\left(B\right.$ and $R$ ) and near-infrared (NIR, $J$ and $K_{\mathrm{s}}$ ) with a cadence of two days. The light curves show unprecedented variability details. The NIR variation features of WPVS48 are consistent with the corresponding optical variations, but the features appear sharper in the NIR than in the optical, suggesting that the optical photons undergo multiple scatterings. The $J$ and $K_{\mathrm{s}}$ emission, tracing the hot $(\sim 1600 \mathrm{~K})$ dust echo, lags the $B$ and $R$ variations by on average $\tau=64 \pm 4$ days and $71 \pm 5$ days, respectively (restframe). WPVS48 lies on the known $\tau-M_{V}$ relationship. However, the observed $\operatorname{lag} \tau$ is about three times shorter than expected from the dust sublimation radius $r_{\text {sub }}$ inferred from the optical-UV luminosity, and explanations for this common discrepancy are searched for. The sharp NIR echos argue for a face-on torus geometry and allow us to put forward two potential scenarios: 1) as previously proposed, in the equatorial plane of the accretion disk the inner region of the torus is flattened and may come closer to the accretion disk. 2) The dust torus with inner radius $r_{\text {sub }}$ is geometrically and optically thick, so that the observer only sees the facing rim of the torus wall, which lies closer to the observer than the torus equatorial plane and therefore leads to an observed foreshortened lag. Both scenarios are able to explain the factor three discrepancy between $\tau$ and $r_{\text {sub. }}$. Longer-wavelength dust reverberation data might enable one to distinguish between the scenarios.
\end{abstract}

Key words. galaxies: active - galaxies: Seyfert - galaxies: individual: WPVS48

\section{Introduction}

In the standard paradigm, active galactic nuclei (AGNs) are composed of a central black hole with an accretion disk (AD). A broad line region (BLR) surrounds the AD, and farther out there is a (clumpy) molecular dust torus (Antonucci 1993). These components cannot be spatially resolved by conventional imaging, but reverberation mapping (RM, Blandford \& McKee 1982) can separate them. In RM, continuum brightness variations of the AD are echoed by the BLR emission lines and by the torus dust emission. The time delay $\tau$ gives the characteristic size ( $R=\tau \cdot c$, where $c$ is the speed of light) and in favorable cases the geometry of the respective regions.

The geometry of AGN dust distributions is still under debate. Dust RM of Seyfert 1 galaxies (Glass 2004; Minezaki et al. 2004; Suganuma et al. 2006) revealed a relation $R_{\mathrm{in}}=\tau \cdot c \propto L_{\mathrm{UV}}^{0.5}$ between the inner radius $R_{\text {in }}$ of the dust torus and the UV luminosity $L_{\mathrm{UV}}$ (Oknyanskij et al. 1999; Kishimoto et al. 2007). For a bagel-shaped dust torus, however, the observed $R_{\text {in }}$ is about a factor three smaller than the sublimation radius $R_{\text {sub }}$ predicted from the dust sublimation temperature $T_{\text {sub }} \sim 1600 \mathrm{~K}$ (Barvainis 1992). Some modified dust geometries involve BLR associated dust due to winds/outflows from the accretion disk (Konigl \& Kartje 1994; Elitzur \& Shlosman 2006; Czerny \& Hryniewicz 2011). Non isotropic illumination of the torus by the AD has

\footnotetext{
* Light curves are only available at the CDS via anonymous ftp to cdsarc.u-strasbg.fr (130.79.128.5) or via http://cdsarc.u-strasbg.fr/viz-bin/qcat?J/A+A/561/L8
}

been proposed as well (Kawaguchi \& Mori 2010), allowing the dust to come closer in the AD's equatorial plane without being sublimated. On the other hand, the $3 \mu \mathrm{m}$ emission bump in the spectral energy distribution of type $1 \mathrm{AGN}$ might be attributed to a puffed-up inner rim of the torus (Krolik 2007).

WPVS48 is a nearby Seyfert- 1 galaxy $(z=0.0377)$ located at a distance of $161 \mathrm{Mpc}$ (Véron-Cetty \& Véron 2010). Singleepoch UBVRI multi-aperture photometry by Winkler (1997) showed evidence of a luminous nucleus $(V=14.78)$. Here we present results for the dust torus geometry of WPVS48. Based on a dust RM campaign, we determine the inner size of the dust torus and the host-subtracted AGN luminosity. The sharp nearinfrared (NIR) variation in WPVS48 allow us to favor two geometric models that are able to explain the factor of three discrepancy between $R_{\text {in }}=\tau \cdot c$ and $R_{\text {sub }}$ in Seyfert 1 galaxies.

\section{Observations and data}

The optical Johnson broad-band $B(4330 \AA)$ and $R(7000 \AA)$ observations were carried out between January 12 and May 13 in 2013, with the robotic $25 \mathrm{~cm}$ Berlin Exoplanet Search Telescope-II (BEST-II), located at the Universitätssternwarte Bochum, near Cerro Armazones, Chile ${ }^{1}$. As in Pozo Nuñez et al. (2013), we performed standard data reduction including corrections for bias, dark current, flatfield, astrometry and astrometric distortion before combining the nine dithered images of each

1 http://www . astro.ruhr-uni-bochum.de/astro/oca/ 

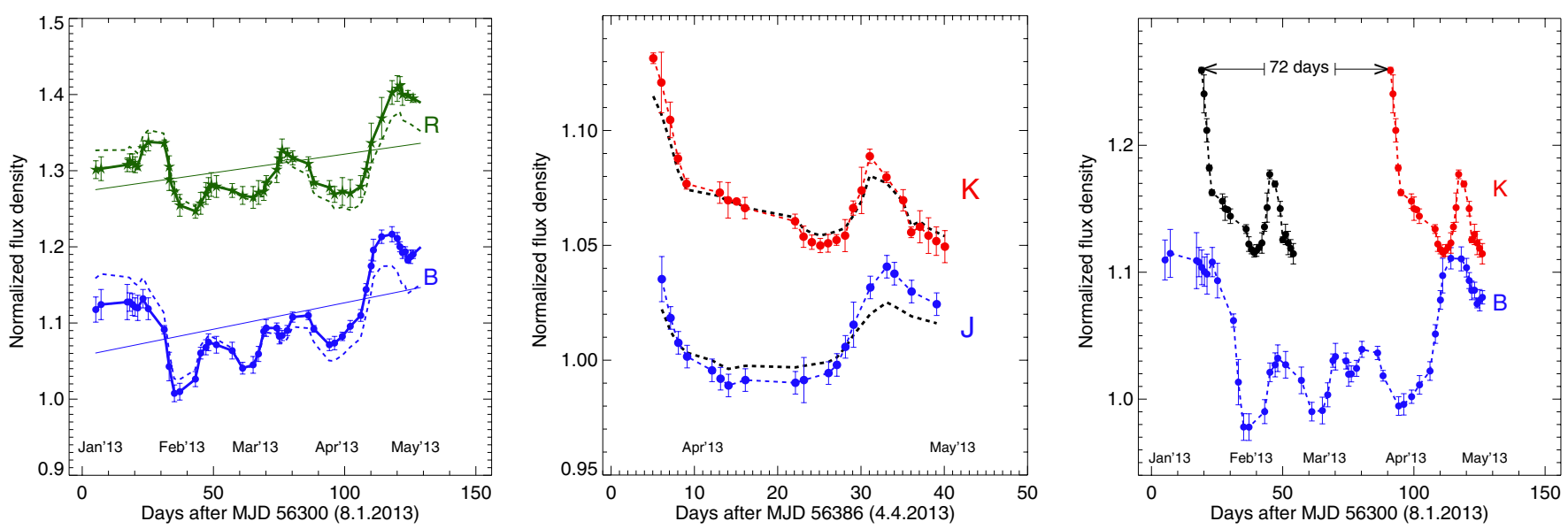

Fig. 1. Left: $B$ - and $R$-band light curves obtained in 2013 January-May. The $R$ light curve is vertically shifted for clarity. The straight lines indicate the long-term brightness trends. The dotted lines show the detrended light curves. Middle: $J$ - and $K$-band light curves obtained in 2013 April-May. The $K$ light curve is vertically shifted for clarity. The dotted light curves depict the state before host galaxy subtraction. Right: $B$ - and $K$-band light curves (blue and red) after host galaxy subtraction. The $K$-band variation pattern (red), when shifted back by $\sim 72$ days (black), largely corresponds to the $B$-band variation pattern in February 2013; see text for details.

night and filter. A 7'.5 diameter aperture was used to extract the photometry and to create flux-normalized light curves relative to 17 non variable stars located in the same images, within $30^{\prime}$ around the AGN, and of similar brightness as the AGN. Absolute calibration was performed using standard reference stars from Landolt (2009) observed on the same nights as the AGN. We also corrected for atmospheric (Patat et al. 2011) and Galactic foreground extinction (Schlafly \& Finkbeiner 2011).

After observing the pronounced optical variations in February 2013, we performed the NIR $J$ and $K_{\mathrm{s}}$ (hereafter denoted as $K$ ) observations between April 10 and May 13 in 2013 using the $0.8 \mathrm{~m}$ Infrared Imaging System (IRIS) telescope (Hodapp et al. 2010) at the Universitätssternwarte Bochum. Images were obtained and reduced in the standard manner. Photometry was extracted using a 7.'0 diameter aperture, slightly smaller than for the optical light curves, because the seeing in the NIR images is slightly better and smaller pixel sizes were used. Light curves were calculated relative to six non variable stars located in the same field that have a similar brightness as the AGN. The photometric calibration was achieved using four high-quality flag (AAA) 2MASS stars in the same field as the AGN. Absolute fluxes were corrected for Galactic foreground extinction (Schlafly \& Finkbeiner 2011).

\section{Results and discussion}

\subsection{Optical and infrared light curves}

The light curves of WPVS48 are shown in Fig 1. A summary of the photometric results and the fluxes in all bands is listed in Table 1. The light curves are published at the CDS. The two optical and the two NIR light curves show a similar variability behavior. The optical light curves exhibit a long-term trend that we corrected for, following Welsh (1999) and Denney et al. (2010), to avoid a potential bias in the cross-correlation analysis. The NIR light curves are too short for detrending.

Using the flux variation gradient (FVG) method (Choloniewski 1981; Winkler et al. 1992; Glass 2004; Sakata et al. 2010), we determined the host galaxy contribution to the light curves (Fig. 2). In brief, it was observationally established that the optical and NIR flux ratios $(B / R$ and $J / K)$ of the variable component remain constant with time, allowing us to separate
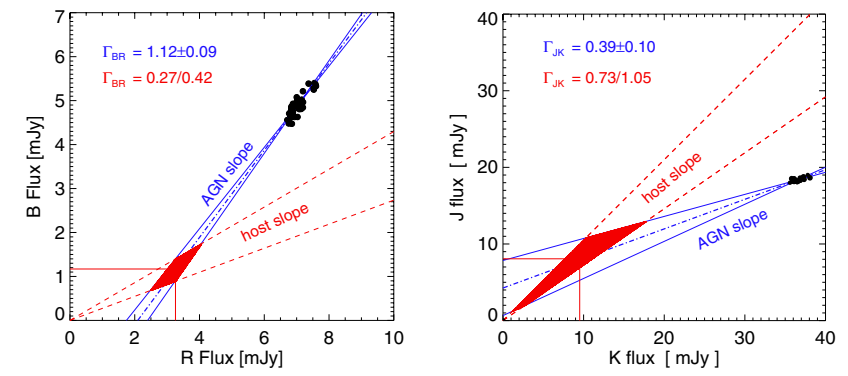

Fig. 2. Flux variation gradient diagrams of WPVS48 in the optical (left) and NIR (right). The data are represented by the black dots. The solid blue lines represent the bisector fit, yielding the range of the AGN slopes. The dashed red lines indicate the range of host slopes determined in the optical by Sakata et al. (2010) and the NIR by Suganuma et al. (2006). The intersection between the host galaxy and AGN slope (red area) gives the host galaxy flux in the respective bands.

the AGN flux through the use of a well-defined range of host galaxy colors (Glass 2004; Sakata et al. 2010). More details on the FVG method are given in Pozo Nuñez et al. (2012). As noted by Glass (2004), the $K$-band light curve may be contaminated by slower-varying longer-wavelength ( $L$ band) emission, and we address this effect in Sect. 3.2. Power-law extrapolation of the AGN $B$ and $R$ fluxes (presumably from the $\mathrm{AD}$ ) to longer wavelengths shows that the contribution of the AD to the $J$ and $K$ fluxes is negligible $(<10 \%)$.

We correlated both the $B$ - and $K$-band light curves and the $B$ - and $J$-band light curves using the discrete correlation function (DCF, Edelson \& Krolik 1988). The DCF centroid yields a time delay $\tau=65.8$ days of $B / J$ and $\tau=72.1$ days of $B / K$ (Fig. 3). The uncertainties of $\tau$ were calculated using the flux randomization and random subset-selection method (FR and RSS, Peterson et al. 2004). The median of this procedure yields $\tau_{\text {cent }}=66.5_{-4.1}^{+3.8}$ days and $\tau_{\text {cent }}=73.5_{-5.2}^{+4.4}$ days for $B / J$ and $B / K$, respectively. Correcting for the time delation factor, we obtain a rest frame time-delay $\tau_{\text {rest }}=64.1 \pm 3.81$ days and $\tau_{\text {rest }}=70.8 \pm 4.63$ days for $B / J$ and $B / K$, respectively.

When the $K$ light curve is shifted back by 72 days (black curve in Fig. 1, right), the variation features (the steep decline, the valley, and the subsequent peak) are roughly consistent with the $B$ features. However, the $K$ decline precedes the $B$ decline, 

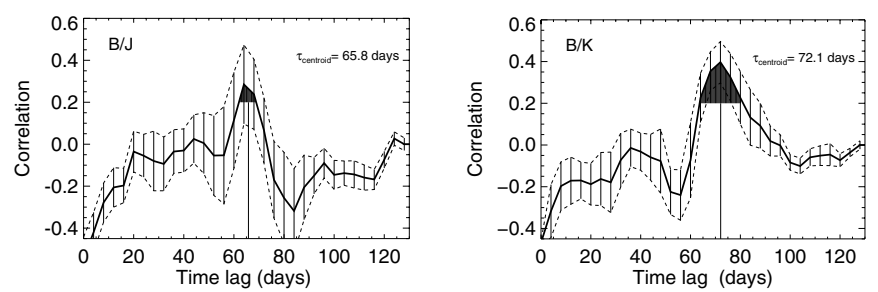

Fig. 3. Discrete correlation function of the $B$ and $J$ and the $B$ and $K$ light curves. The dotted lines indicate the error range $( \pm 1 \sigma)$ around the cross correlation. The black shaded area marks the range used to calculate the centroid of the lag (vertical line).

the subsequent peak is sharper in $K$ than in $B$, and the time span of the $K$ valley (between the decline and the subsequent peak) is longer than for $B$. The same holds for the $R$ and $J$ curves, respectively. An explanation (private communication by Ralf Siebenmorgen and Endrik Krügel; see also Krügel 2009) might be that on the way to the observer a significant fraction of the optical photons are scattered multiple times in the $\mathrm{kpc}-$ scale narrow-line region. This leads to a longer traveling path to the observer, hence a delayed arrival. The net effect is that we observe the $\mathrm{AD}$ variability convolved with an asymmetric delay kernel. The longer-wavelength NIR photons may be scattered in the torus, but with small path-length changes, and they are only weakly affected by scattering in the NLR, so that their observed variation features remain sharp, even sharper than the optical features.

One might expect that AD brightening leads to dust destruction and the receding of the dust sublimation front, and vice versa. Then after a bright NIR light curve plateau a decline should be delayed and a rise after a low state should occur earlier, but the variations of the current NIR data are too small to allow an identification of such signatures.

Dust-reverberation studies of ten Seyfert 1 galaxies have shown that the lag $\tau$ of the dust $K$-band emission is proportional to the square root of the optical luminosity ( $\tau \propto L^{0.5}$; Suganuma et al. 2006 and references therein). We interpolated $M_{V}$ of WPVS48 from our $B$ and $R$ data. Figure 4 shows its position in the $\tau-M_{V}$ diagram close to the regression line.

\subsection{Inner geometry of the dust torus}

Photometric $\mathrm{H} \alpha$ reverberation-mapping of WPVS48 revealed that the echo of the H $\alpha$ BLR has a mean lag of 25 days (Pozo Nuñez et al. in prep). In consequence, because of the different lags of $\mathrm{H} \alpha$ and dust echo, BLR-related dust probably plays a minor role.

If the dust distribution is spread far along the line-of-sight, one would expect that the echo is smeared out in time. Therefore the sharp dust echo of WPVS48 argues in favor of a face-on torus geometry. We focused on the basic geometry of the overall absorbing dust, and considered smooth dust distributions. The topic of a clumpy dust structure is left for future modeling.

A general puzzle from dust RM is that with reasonable assumptions for graphite grains of a sublimation temperature $\sim 1600 \mathrm{~K}$ and size $0.05 \mu \mathrm{m}$ in radius (Barvainis 1992) and for a bagel-shaped torus, the inner radius $R_{\text {in }}=\tau \cdot c$ is about three times smaller than the dust sublimation radius $r_{\text {sub }}$ inferred from the optical-UV luminosity (Kishimoto et al. 2007).

To solve this puzzle, Kawaguchi \& Mori (2010, hereafter KM2010, 2011) have considered a model where the accretion disk emits relatively little radiation in its equatorial plane. The

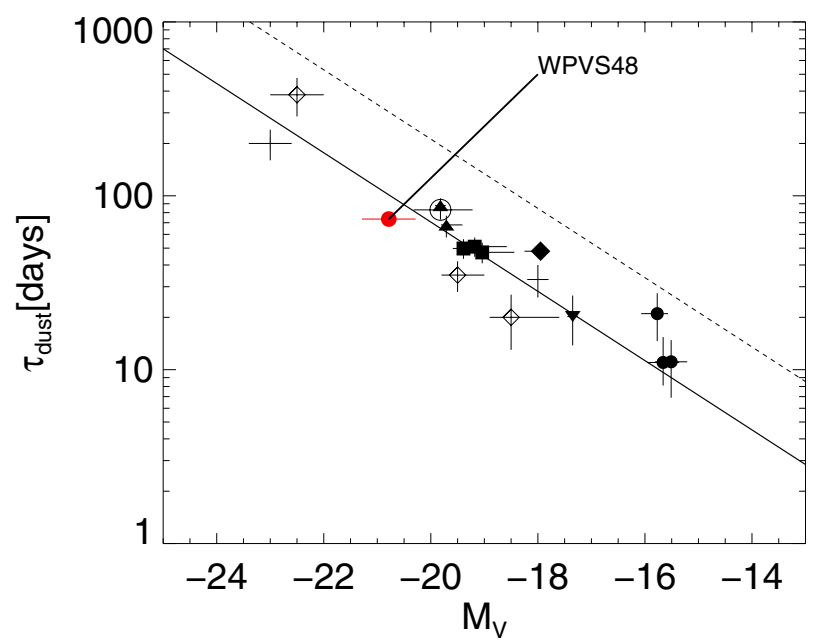

Fig. 4. Lag-luminosity relationship based on the data of Suganuma et al. (2006). The symbols for individual Seyfert 1 galaxies are the same as in Suganuma et al. (2006). The solid line is the best-fit regression from Suganuma et al. (2006). WPVS48 (red dot) lies close to the regression line. The dashed line indicates the dust sublimation radius $r_{\text {sub }}$ expected at a given nuclear luminosity $M_{V}$ (from Kishimoto et al. 2007).

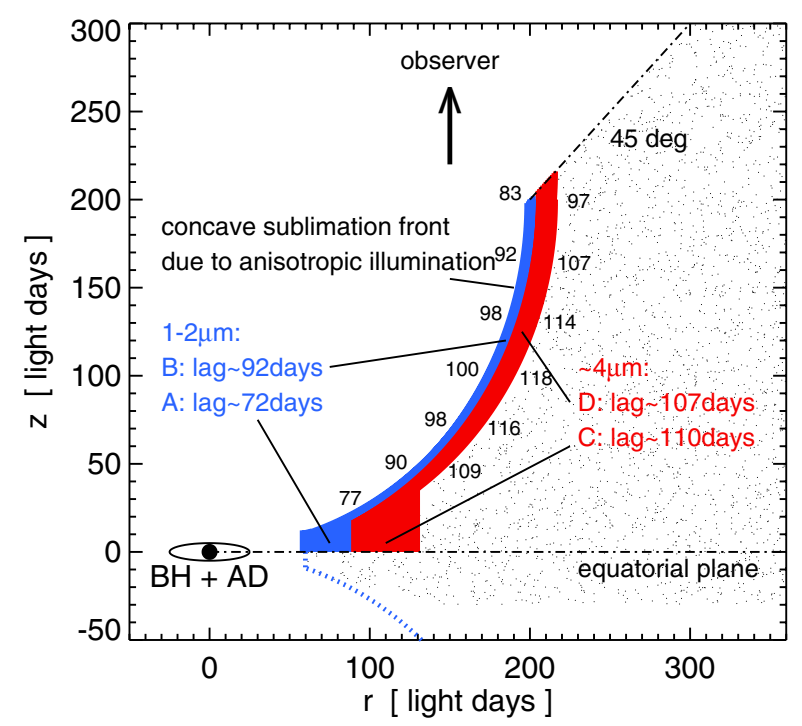

Fig. 5. Schematic illustration of the dust torus model by Kawaguchi \& Mori. Blue denotes hot $T \sim 1600 \mathrm{~K}$ dust visible at $1-2 \mu \mathrm{m}$, red denotes cooler $T \sim 800 \mathrm{~K}$ dust visible at $\sim 4 \mu \mathrm{m}$. The single black numbers left and right of the rim give the lags along the torus rim at $1-2 \mu \mathrm{m}$ and at $\sim 4 \mu \mathrm{m}$, respectively. Note the foreshortening of the lags in the highaltitude part of the torus rim. The mean lags of the equatorial zones (A and C) and the entire $2 \pi$ integrated torus rim (B and D) are given, adopting a constant dust density distribution along the rim.

anisotropic illumination allows the torus inner region in the equatorial plane to approach to the central black hole and shapes the inner torus wall so that it is concave from the observers view, with the height $z$ above the equatorial plane increasing radially with $r$ to a covering half-angle of $\sim 45^{\circ}$. In this picture, illustrated in Fig. 5, the hot dust emission comes from a geometrically thin inner dust torus with a remarkably small dust covering angle, and along the dust sublimation rim.

Specifically for WPVS48, the dust may approach in the AD's equatorial plane, without sublimation, and the hot dust will produce a sharp echo at mean lag $\sim 72$ days, but the long inner torus rim will add a smeared-out echo of a longer lag ranging between $80 \mathrm{~d}>\tau<100 \mathrm{~d}$ (Fig. 5). The bulk of the dust 


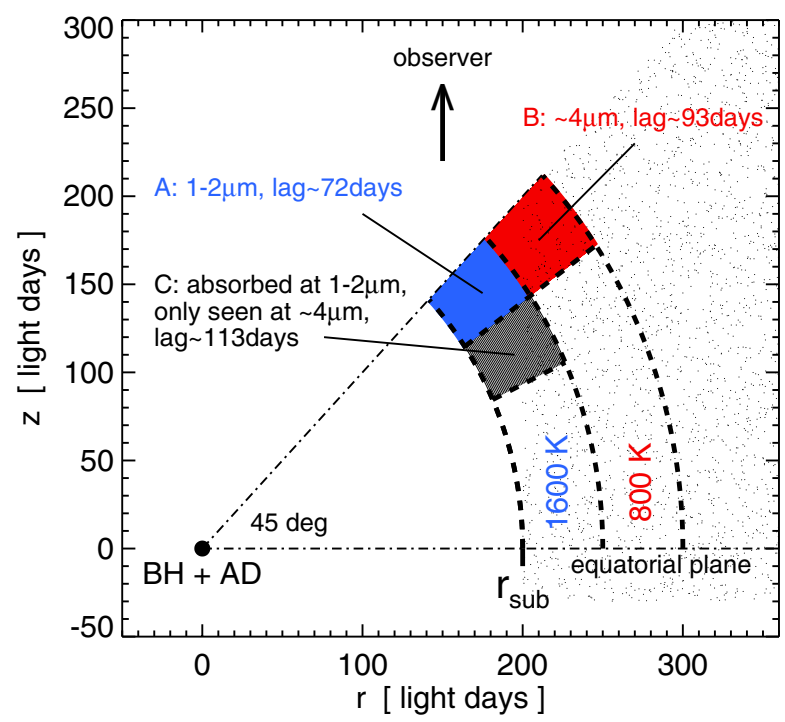

Fig. 6. Schematic illustration of a torus with constant sublimation radius for inclinations $0^{\circ}<i<45^{\circ}$, optically thick at $1-2 \mu \mathrm{m}$. Zone A (blue) marks hot $T \sim 1600 \mathrm{~K}$ dust visible at $1-2 \mu \mathrm{m}$, zone B (red) cooler $T \sim 800 \mathrm{~K}$ dust visible at $\sim 4 \mu \mathrm{m}$; zone $\mathrm{C}$ (shaded black) marks hot dust that is heavily obscured $\left(A_{K}>1\right)$ in the observer's line of sight, so that it becomes dim at $1-2 \mu \mathrm{m}$, but is clearly visible at $\sim 4 \mu \mathrm{m}$. The mean lags for each zone are given.

echo of WPVS48 is as sharp as the triggering continuum variations in the $J$ and $K$ light curves with a rapid decline in $J$ and a two-step (rapid and subsequent shallow) decline in $K$ (Fig. 1 , middle). In the cross correlation $B / K$ a faint tail clearly extends to $\sim 100$ days, but not in $B / J$ (Fig. 3 ). Therefore the current data of WPVS48 appear to be not fully consistent with the expectations from the KM2010 model: Firstly, if the dust sublimation front is shaped by the anisotropic illumination from the AD alone, as proposed by KM2010, one would expect that the hottest $J$ - emitting dust exists along the entire rim and that its signatures seen in the $K$ light curve and the $B / K$ cross correlation are also seen in $J$. Secondly, this interpretation of the slow shallow $K$ decline leaves no room for $K$-band variations that are associated in part with longer-wavelength $L$-band flux (Glass 2004). Thirdly, while, in addition to the radiation fields, AGN winds might help to shape the torus rim, the geometry of the AD (thin disk vs. disk becoming thicker with increasing radius) is unknown, and it is not clear how strong the anisotropy actually is (e.g. Antonucci 2013 and references therein).

We here also considered an alternative scenario, illustrated in Fig. 6. In this picture, for simplicity, we assumed that the AD essentially radiates isotropically. The dust torus is geometrically thick with inner radius $r_{\text {sub }}$ and an opening half angle $\sim 45^{\circ}$ and an optically thick at NIR wavelengths, so that the observer only sees the facing rim of the entire inner dust torus wall. The striking feature is that the rim lies at height $z$, that is, closer to the observer than the equatorial plane. This $z$ contribution foreshortens the observed lag, namely $\tau \cdot c=\left(r^{2}+z^{2}\right)^{0.5}-z \approx r_{\text {sub }}-z$. If the rim lies at an angle $45^{\circ}$ from the equatorial plane, one obtains $z=\cos \left(45^{\circ}\right) \cdot r_{\text {sub }}$, hence $\tau \cdot c=0.3 \cdot r_{\text {sub }}$. This scenario can explain the factor three discrepancy between $\tau$ and $r_{\text {sub }}$ as an observational foreshortening effect. The $J$ and $K$ light curves are consistent with this picture: the rapid decline in $J$ and $K$ arises from dust in the blue zone of Fig. 6 and the subsequent shallow decline in the $K$ light curve might be explained by dust in the transition zone between the blue zone and the red and black zones, which are not warm enough (red zone) or too much
Table 1. Photometry range in mag and mean flux densities with errors in $\mathrm{mJy}$.

\begin{tabular}{cccc}
\hline \hline$B$ & $R$ & $J$ & $K$ \\
\hline $14.74-14.94$ & $13.93-14.06$ & $12.33-12.36$ & $10.61-10.66$ \\
\hline$f B$ & $f R$ & $f J$ & $f K$ \\
\hline $4.90 \pm 0.04$ & $7.04 \pm 0.07$ & $18.36 \pm 0.46$ & $36.56 \pm 1.10$ \\
\hline
\end{tabular}

Notes. The values are as observed in 7'. $5(B, R)$ and 7.'0 $(J, K)$ apertures. absorbed (black zone) in $J$. Then the shallow $K$ decline would be associated with longer-wavelength $L$-band flux, noted in other sources by Glass (2004).

Future dust RM including longer wavelengths (e.g. at 3.6 and $4.5 \mu \mathrm{m}$ ) may provide clues to distinguish between the two models. Firstly, the contribution of $T \sim 800 \mathrm{~K}$ cool dust, visible at $4 \mu \mathrm{m}$, to the $K$ band can be constrained. Secondly, for the Kawaguchi \& Mori model (Fig. 5), the expected $4 \mu \mathrm{m}$ lags are similar along the rim (component D) and in the equatorial plane (component C). On the other hand, for the geometrically and optically thick torus model (Fig. 6), the cool dust (component B) and the hot dust with high extinction (component C), as seen by the observer, might produce different lags of equal strength, resulting in light curves with a two-step behavior.

Acknowledgements. This work is supported by the Nordrhein-Westfälische Akademie der Wissenschaften und der Künste in the framework of the academy program of the Federal Republic of Germany and the state Nordrhein-Westfalen, by Deutsche Forschungsgemeinschaft (DFG HA3555/12-1) and by Deutsches Zentrum für Luft und Raumfahrt (DLR 50 OR 1106).

We thank our referee Ian Glass for helpful comments and careful review of the manuscript. We warmly thank Endrik Krügel and Ralf Siebenmorgen for discussions. The observations on Cerro Armazones benefitted from the care of guardians Hector Labra, Gerardo Pino, Roberto Munoz, and Francisco Arraya. This research has made use of SIMBAD and the NASA/IPAC Extragalactic Database (NED).

\section{References}

Antonucci, R. 1993, ARA\&A, 31, 473

Antonucci, R. 2013, Nature, 495, 165

Barvainis, R. 1992, ApJ, 400, 502

Blandford, R. D., \& McKee, C. F. 1982, ApJ, 255, 419

Choloniewski, J. 1981, Acta Astron., 31, 293

Czerny, B., \& Hryniewicz, K. 2011, A\&A, 525, L8

Denney, K. D., Peterson, B. M., Pogge, R. W., et al. 2010, ApJ, 721, 715

Edelson, R. A., \& Krolik, J. H. 1988, ApJ, 333, 646

Elitzur, M., \& Shlosman, I. 2006, ApJ, 648, L101

Glass, I. S. 2004, MNRAS, 350, 1049

Hodapp, K. W., Chini, R., Reipurth, B., et al. 2010, Proc. SPIE, 7735, 1A

Kawaguchi, T., \& Mori, M. 2010, ApJ, 724, L183

Kawaguchi, T., \& Mori, M. 2011, ApJ, 737, 105

Kishimoto, M., Hönig, S. F., Beckert, T., \& Weigelt, G. 2007, A\&A, 476, 713

Konigl, A., \& Kartje, J. F. 1994, ApJ, 434, 446

Krolik, J. H. 2007, ApJ, 661, 52

Krügel, E. 2009, A\&A, 493, 385

Landolt, A. U. 2009, AJ, 137, 4186

Minezaki, T., Yoshii, Y., Kobayashi, Y., et al. 2004, ApJ, 600, L35

Oknyanskij, V. L., Lyuty, V. M., Taranova, O. G., \& Shenavrin, V. I. 1999, Astron. Lett., 25, 483

Patat, F., Moehler, S., O’Brien, K., et al. 2011, A\&A, 527, A91

Peterson, B. M., Ferrarese, L., Gilbert, K. M., et al. 2004, ApJ, 613, 682

Pozo Nuñez, F., Ramolla, M., Westhues, C., et al. 2012, A\&A, 545, A84

Pozo Nuñez, F., Westhues, C., Ramolla, M., et al. 2013, A\&A, 552, A1

Sakata, Y., Minezaki, T., Yoshii, Y., et al. 2010, ApJ, 711, 461

Schlafly, E. F., \& Finkbeiner, D. P. 2011, ApJ, 737, 103

Suganuma, M., Yoshii, Y., Kobayashi, Y., et al. 2006, ApJ, 639, 46

Véron-Cetty, M.-P., \& Véron, P. 2010, A\&A, 518, A10

Welsh, W. F. 1999, PASP, 111, 1347

Winkler, H. 1997, MNRAS, 292, 273

Winkler, H., Glass, I. S., van Wyk, F., et al. 1992, MNRAS, 257, 659 